\title{
La situation économique des personnes âgées et les impacts du vieillissement sur la structure de peuplement : Le cas du Bas-Saint-Laurent
}

\author{
Majella Simard \\ Université de Moncton
}

\section{Introduction}

L'évolution du niveau de vie des aînés constitue l'une des principales mutations qu'a connues la société québécoise au cours des dernières décennies. De 1981 à 2001, au Québec, la croissance du revenu moyen des aînés a été plus importante que pour celle des familles. Cette croissance a effectivement été de $278,1 \%$ pour celles-ci en comparaison à $293,4 \%$ pour ceux-là. Cette situation témoigne du succès des politiques en matière de prestations et de fiscalité. Durant cette période, les transferts gouvernementaux à destination des personnes âgées ont connu une augmentation substantielle que ce soit par le biais des prestations de la sécurité de la vieillesse, du supplément de revenu garanti, du régime de pensions du Canada et du régime de rentes du Québec. Bien que le revenu des personnes âgées du Bas-SaintLaurent se soit considérablement amélioré au cours des 20 dernières années, la persistance de profondes disparités constitue l'un des principaux éléments de spécificité qui démarque de façon particulière cette région.

L'objectif de cet article consiste à examiner l'évolution des disparités économiques des personnes âgées sur la base de leur revenu moyen au sein de la région du Bas-SaintLaurent. Nous orienterons plus particulièrement notre analyse sur les localités rurales, c'est-à-dire celles de 2500 habitants et moins. Cet examen sera effectué en considérant la méthode des strates démographiques et la typologie du ministère des Affaires municipales et des Régions. Celui-ci, à partir d'un indice synthétique de développement, a élaboré, en 1996, une typologie à quatre niveaux illustrant autant de situations socioéconomiques. Cet indice intégrait huit indicateurs, à savoir: la variation de la population entre 1991 et 1996, le rapport emploi/population, le pourcentage d'individus dont la scolarité est inférieure à la $9^{\mathrm{e}}$ année, le taux de chômage, le pourcentage du revenu perçu sous la forme de transferts gouvernementaux, la fréquence des faibles revenus et le revenu moyen des ménages. Nous avons repris la typologie du ministère en l'adaptant aux données du recensement de 2001. Ainsi, à l'échelle du Bas-Saint-Laurent, les quatre catégories de localités ont été déterminées de la manière suivante: 
Tableau 1

Typologie des localités rurales du Bas-Saint-Laurent telle que déterminée par le ministère des Affaires municipales et des Régions (version 2001)

\begin{tabular}{|l|c|c|c|}
\hline \multicolumn{1}{|c|}{ Catégories de localités } & Indice de développement & Nombre de localités & \% \\
\hline en restructuration & $-5,00$ et moins & 60 & 54,1 \\
\hline stables & $-4,99$ à 0 & 34 & 30,6 \\
\hline dynamiques & 0 à 4,99 & 15 & 13,5 \\
\hline très dynamiques & 5,00 et plus & 2 & 1,8 \\
\hline
\end{tabular}

Source : Banque de données fournies par le ministère des Affaires municipales et des Régions.

La « gérontocroissance » et le

vieillissement constituent un fait bien réel au Bas-Saint-Laurent.

À l'instar de ce que nous observons à l'échelle du Québec, la «gérontocroissance » (caractérisée par l'augmentation du nombre de personnes âgées) et le vieillissement (c'està-dire l'accrois-sement des aînés en valeur relative) constituent un fait bien réel au BasSaint-Laurent. Mais quels sont les impacts de ce double processus sur la structure de peuplement régional? Nous tenterons de répondre à cette question dans la deuxième partie de cette contribution.

\section{La situation économique des personnes âgées en 2001}

Analyse globale

D'entrée de jeu, nous constatons au graphique 1 que le revenu moyen des personnes âgées, au Bas-Saint-Laurent, est inférieur à celui de la province. La différence est de l'ordre de 2710 \$. Les disparités ne se manifestent pas seulement

entre la région et la province, mais aussi entre les milieux ruraux et urbains, entre les différentes catégories de milieux ruraux et, bien évidemment, entre les individus.Par ailleurs, l'écart qui sépare le revenu moyen des personnes âgées vivant en milieu rural comparativement à ceux qui habitent la ville est beaucoup moins élevé au Bas-SaintLaurent (1 314 \$) que pour l'ensemble de la province (2 $929 \$$ ). De toute évidence, il appert que les aînés du Bas-Saint-Laurent sont moins confrontés aux inégalités infrarégionales que leurs congénères québécois.

Il n'en demeure pas moins qu'il persiste des écarts considérables notamment entre les différentes catégories de milieux ruraux, comme en fait foi le graphique 2. Les disparités les plus criantes se manifestent au sein des plus petites localités, dont le niveau de revenu des aînés ne correspond qu'à $78,6 \%$ de celui de la province. En outre, une corrélation parfaitement linéaire se dessine entre la situation socio-économique d'une localité, telle que mesurée par le ministère des Affaires municipales et des Régions, et le niveau de revenu des personnes âgées. De fait, celui-ci passe de $15014 \$$ dans les milieux en restructuration à $17208 \$$ pour les localités très dynamiques. Toutefois, ce revenu, au Bas-Saint-Laurent, reste partout inférieur à la moyenne provinciale ce qui n'est pas le cas à l'échelon du Québec rural puisque le revenu moyen des aînés vivant au sein des localités très dynamiques était, en 2001, de $3457 \$$ supérieur à celui de la province. 
Graphique 1

Revenu moyen des personnes âgées de 65 ans ou plus au sein des milieux ruraux et urbains du Bas-Saint-Laurent en comparaison avec la province en 2001

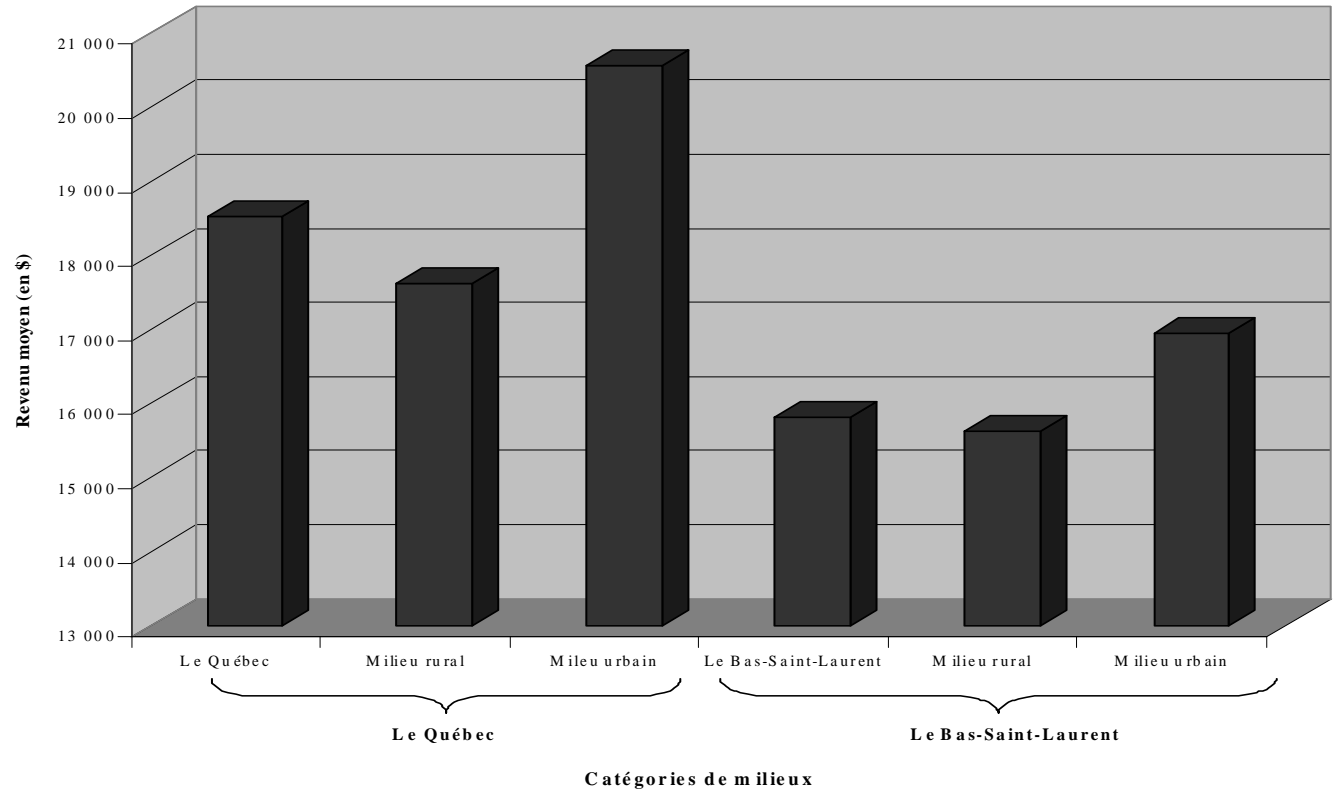

Sources : Statistique Canada. Recensement de 2001.

Graphique 2

Revenu moyen des personnes âgées de 65 ans ou plus au Bas-Saint-Laurent en comparaison avec celui de la province pour différentes catégories de localités rurales en 2001

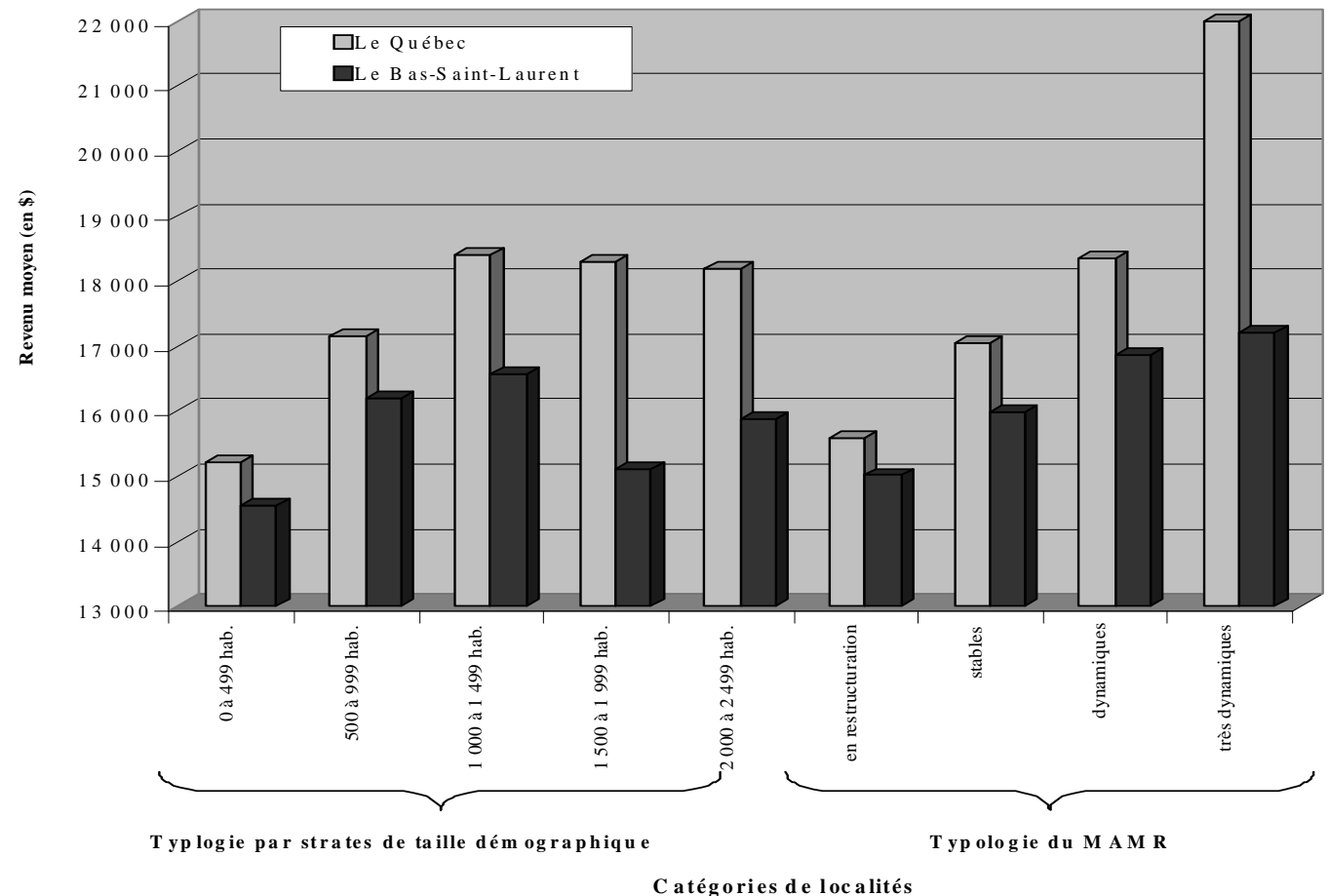

Source : Statistique Canada. Recensement de 2001. 


\section{Disparités locales de revenu}

Bien que très éloquents, les écarts de revenus entre les milieux ruraux du Bas-Saint-Laurent et ceux de la province ne traduisent qu'une vision fragmentaire des disparités. Celles-ci apparaissent avec beaucoup plus d'acuité lorsqu'on ramène l'échelle d'analyse à l'échelon local. Nous avons représenté, à la carte 1 , les subdivisions de recensement en fonction du niveau de revenu des personnes âgées de 65 ans ou plus en 2001. Seulement 20 municipalités disposent d'un revenu

Les personnes âgées ne constituent pas la seule catégorie d'individus qui souffrent d'inégalités économiques au sein des milieux fragiles.
Pocatière, Ste-Luce, Pointe-au-Père, Cacouna et Notre-Dame-u-Portage.

À l'opposé, on dénombre 32 localités dont le niveau de revenu des aînés n'équivaut qu'à $75 \%$ ou moins à celui de la province. Ces municipalités sont identifiées par leur toponyme à la carte 1 . L'essentiel de ce segment est constitué de milieux en restructuration. Ces derniers ont tendance forment des blocs contigus notamment dans les moyen supérieur à celui du Québec c'est-àdire à $18 \quad 521 \$$. Parmi celles-ci, six correspondent à des villes alors que six autres se situent à la périphérie de celles-ci ou encore exercent le rôle de village-dortoir. C'est notamment le cas de St-Joseph-deKamouraska qui détient le niveau le plus élevé. Un élément d'explication tient au fait que ces municipalités abritent, de façon générale, un contingent relativement élevé (15 $\%$ ou plus) d'individus ayant complété une formation universitaire. Nous pensons notamment à des localités telles que $\mathrm{La}$
MRC de Matapédia, du Témiscouata et des Basques ce qui illustre bien l'aspect structurel du problème. La présence de Ste-Odile parmi le peloton de queue étonne à plus d'un titre en raison de sa proximité par rapport à Rimouski, principal pôle urbain de la région. Par ailleurs, les personnes âgées ne constituent pas la seule catégorie d'individus qui souffrent d'inégalités économiques au sein des milieux fragiles. En effet, plusieurs de ceux-ci figurent parmi les plus pauvres de la région.

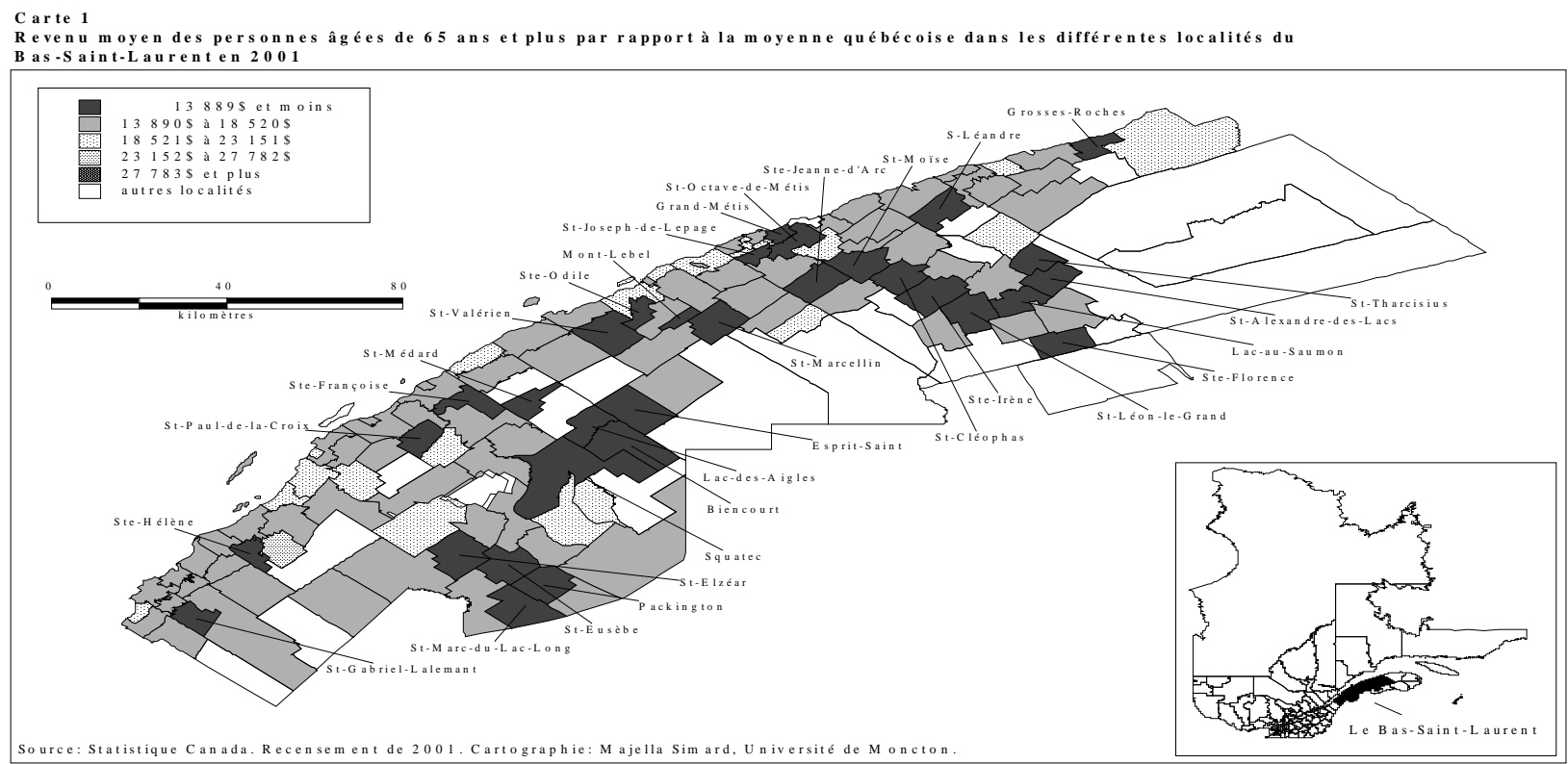




\section{Évolution au cours de la période 1981-2001}

Le fait de considérer le revenu d'une seule année pour identifier les milieux les plus fragiles eu égard au revenu moyen des aînés amène un certain degré d'imprécision. Les niveaux de revenu changent d'une période quinquennale à l'autre. Une analyse comparative sur une échelle temporelle de 20 ans permet de mieux cerner l'évolution des disparités et d'identifier les principaux éléments de différenciation. L'un de ceux-ci tient au fait que le revenu des aînés a augmenté plus rapidement au sein de la province dans trois catégories de localités rurales à savoir celles dont la taille démographique varie entre 500 et 999 habitants, les milieux en restructuration et les municipalités stables (tableau 2). À l'échelle du Québec, seules les petites localités et celles considérées comme dynamiques par le ministère des Affaires municipales et des Régions étaient parvenues à augmenter le niveau de revenu de leurs aînés plus rapidement que celui de la province. Une telle situation est symptomatique de la persistance d'inégalités qui affectent plusieurs segments de l'espace rural au Bas-Saint-Laurent. Mais l'élément le plus frappant que révèle ce même tableau concerne la valeur moyenne d'augmentation du niveau de revenu des aînés que l'on observe en milieu urbain. Cette dernière est nettement inférieure à celle de la province et de l'ensemble du milieu rural baslaurentien. Bref, l'accroissement des disparités est aussi un fait bien présent au sein des villes du Bas-Saint-Laurent.

\section{Tableau 2}

Variation du revenu moyen des personnes âgées et écart par rapport à la moyenne provinciale par différentes catégories de milieux au Bas-Saint-Laurent en comparaison avec la province entre 1981 et 2001

\begin{tabular}{|c|c|c|}
\hline Catégories de milieux & Variation du revenu & $\begin{array}{l}\text { Écart du revenu par rapport à } \\
\text { la moyenne provinciale }\end{array}$ \\
\hline \multicolumn{3}{|c|}{ Typologie par strates de taille démographique } \\
\hline Localités de 0 à 499 habitants & 289,0 & $-4,4$ \\
\hline Localités de 500 à 999 habitants & 321,9 & 28,5 \\
\hline $\begin{array}{lllll}\begin{array}{l}\text { Localités } \\
\text { habitants }\end{array} & \text { de } & 999 & \text { à } & 1499 \\
\end{array}$ & 291,6 & $-1,8$ \\
\hline $\begin{array}{lllll}\begin{array}{l}\text { Localités } \\
\text { habitants }\end{array} & \text { de } & 1500 & \text { à } & 1999 \\
\end{array}$ & 259,4 & $-3,4$ \\
\hline $\begin{array}{lllll}\begin{array}{l}\text { Localités de } \\
\text { habitants }\end{array} & 2000 \text { à } 2499 \\
\end{array}$ & 284,6 & $-8,8$ \\
\hline \multicolumn{3}{|c|}{ Typologie du ministère des Affaires municipales et des Régions } \\
\hline Milieux en restructuration & 294,8 & 1,4 \\
\hline Localités stables & 317,6 & 24,2 \\
\hline Localités dynamiques & 269,3 & $-24,1$ \\
\hline Localités très dynamiques & 268,8 & $-24,6$ \\
\hline Milieu rural & 297,3 & 3,9 \\
\hline Milieu urbain & 281,5 & $-11,9$ \\
\hline Le Bas-Saint-Laurent & 295,1 & 1,7 \\
\hline Milieu rural québécois & 296,8 & 3,4 \\
\hline Milieu urbain québécois & 286,8 & $-6,6$ \\
\hline Le Québec & 293,4 & \\
\hline
\end{tabular}

Source : Statistique Canada. Recensement de 1981 et de 2001. 
En réduisant l'échelle d'analyse à l'échelon local, nous remarquons, à la carte 2 , que seulement six localités se sont distinguées par un accroissement du revenu de leurs aînés supérieur à celui de la province. Les localités dont le revenu moyen des aînés s'est accru de $73,3 \%$ ou moins entre 1981 et 2001 sont au nombre de 23. Elles ont tendance à se regrouper dans les MRC de La Matapédia, du Témiscouata et des Basques. Une telle concentration reflète bien l'aspect structurel du problème.

\section{La majorité des localités du Bas-Saint- Laurent, soit plus précisément 68, se sont distinguées par une réduction des inégalités en ce qui concerne le revenu moyen des aînés au cours de la période 1981-2001.}

En calculant l'écart du revenu moyen des 65 ans ou plus par rapport à la moyenne provinciale, il est possible de déterminer la position de chaque localité et, par conséquent, de mieux cerner l'ampleur des disparités. Ainsi que le montre le tableau 2, cet écart est négatif pour l'ensemble de la région ce qui laisse présager un élargissement des disparités au cours de la période 1981-2001. D’après ce même tableau, le milieu rural bas-laurentien, considéré dans sa globalité, n'aurait pas été atteint par un accroissement des disparités eu égard à ce même indicateur. Toutefois, en adoptant une échelle d'analyse plus fine, nous constatons que ce ne sont pas toutes les localités rurales qui sont concernées par cette embellie. C'est le cas notamment des milieux dynamiques et très dynamiques, mais aussi de ceux dont la taille démographique est supérieure à 999 habitants. Tel qu'en fait foi la carte 3, un examen plus attentif de cet indicateur à l'échelle locale permet de constater une situation pour le moins inquiétante à Ste-Anne-de-La-Pocatière, à StArsène, à Notre-Dame-des-Neiges-de-TroisPistoles, à St-Marc-du-Lac-Long, à Cabano et à Amqui. Dans ces milieux, les disparités de revenus ont pris une ampleur considérable en l'espace de 20 ans. De toute évidence, ils devraient faire l'objet de mesures spécifiques à destination des personnes âgées de façon à ce que leur qualité de vie ne s'amenuise pas davantage. Il n'en demeure pas moins que la majorité des localités du Bas-Saint-Laurent, soit plus précisément 68, se sont distinguées par une réduction des inégalités en ce qui concerne le revenu moyen des aînés au cours de la période 1981-2001.

\section{Les impacts de la « gérontocroissance » et du vieillissement démographique sur la structure de peuplement}

Évaluer les conséquences de la «gérontocroissance» et du vieillissement démographique sur le tissu de peuplement régional n'est pas une mince affaire. Les ressources naturelles et l'économie constituent des éléments qui ont des influences considérables sur la structure de peuplement. De plus, les caractéristiques sociodémographiques de toute population locale sont en constante évolution. À maints endroits, les migrations de retour des personnes âgées prennent de l'ampleur. À cet égard, la retraite joue un rôle déterminant sur l'évolution du tissu de peuplement. Elle encourage les déplacements, lesquels sont motivés par la recherche d'agréments tels que la proximité des services et la qualité de l'environnement physique. 


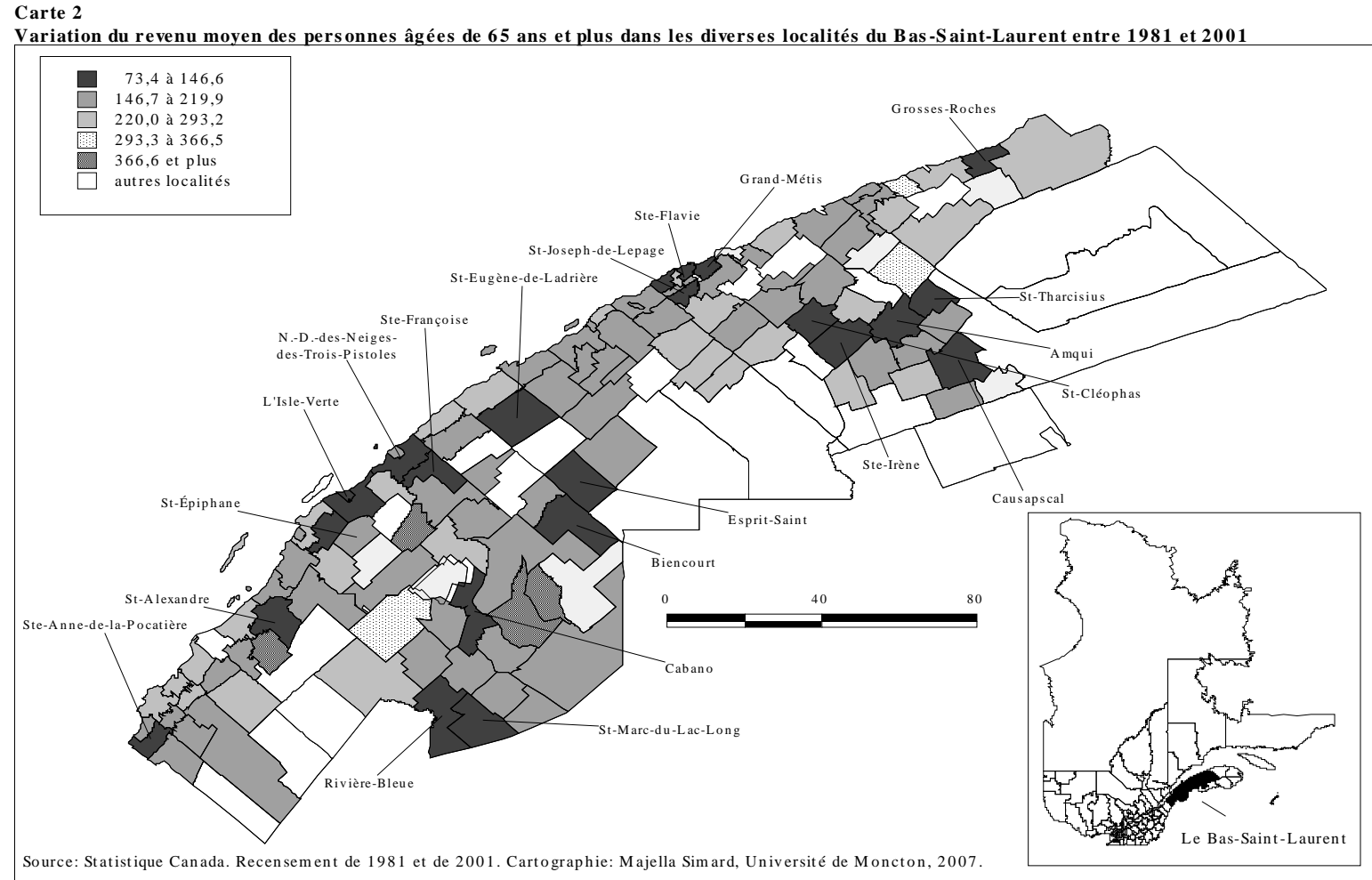

Carte 3

Écart du revenu moyen des personnes âgées de 65 ans et plus par rapport à la moyenne provinciale dans les différentes localités du B as-S aint-Laurent entre 1981 et 2001

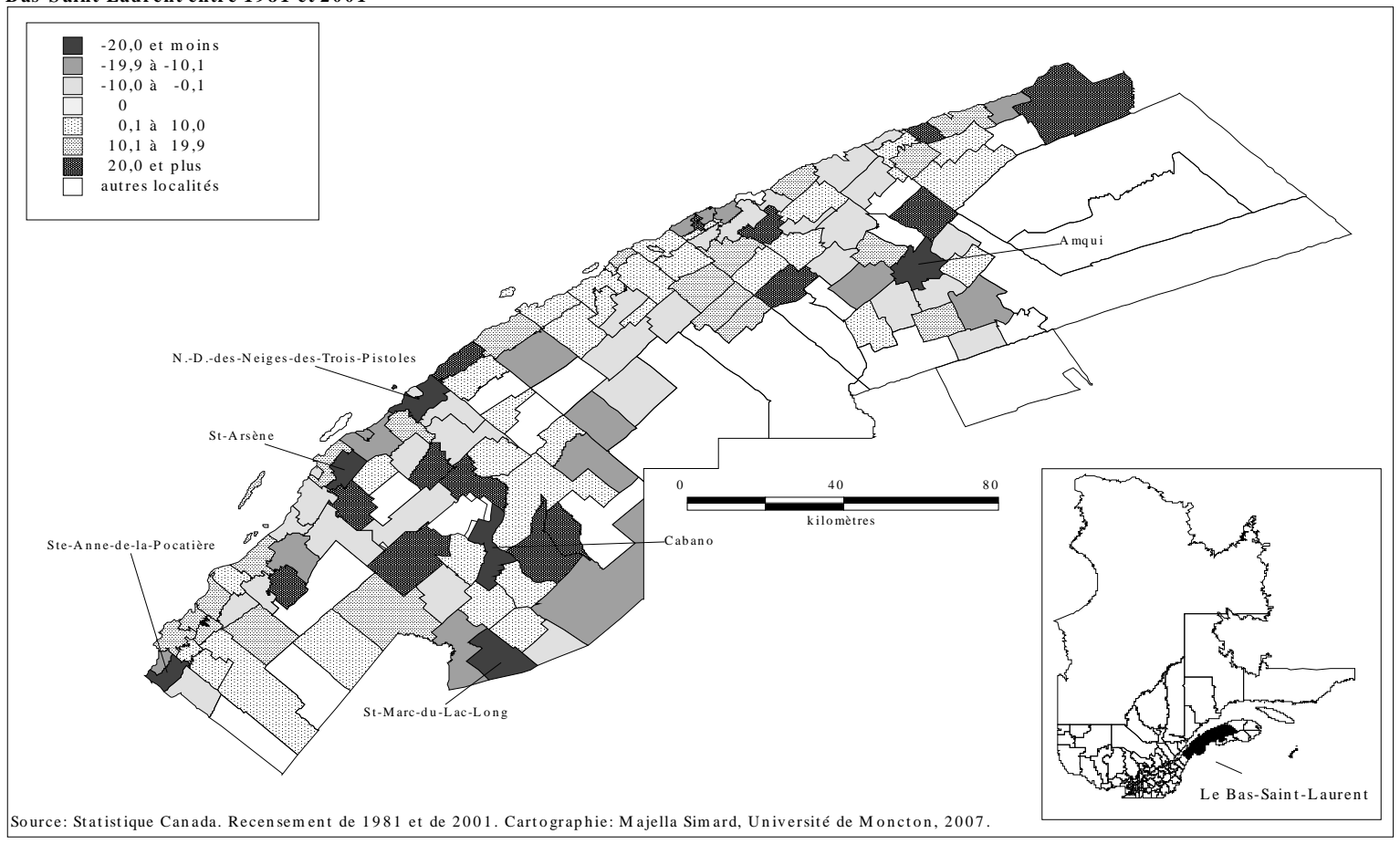

De même, la poursuite des études et la recherche d'un emploi créent de nouvelles séries de pressions sur le semis de peuplement qui favorise généralement les villes au 
détriment des milieux ruraux fragiles. Ainsi, au cours d'une année, il se produit dans presque toutes les collectivités, tant rurales qu'urbaines, un va-et-vient important de jeunes et de personnes âgées. Ce sont les caractéristiques globales de la migration d'entrée et de sortie qui détermineront si ces mouvements auront un effet sur les caractéristiques ou la taille de la population âgée. Si certains milieux connaissent, au BasSaint-Laurent comme ailleurs au Québec, un renouveau, pour l'instant timide, mais non moins significatif, ces migrations de retour contribuent à une redistribution des activités et de la population sur le territoire régional. Par ailleurs, bien malin est celui qui pourra préciser quelle sera l'ampleur véritable de ce phénomène qu'il faudrait d'abord mieux saisir quantitativement pour le comprendre. Ces mobilités ont donc des impacts différents selon les milieux géographiques. Leurs effets peuvent être positifs ou négatifs selon l'échelle, à laquelle on les considère.

\section{Conclusion}

\section{Devant une telle complexité, il nous apparaît particulièrement} opportun et réaliste que les interventions des diverses instances gouvernementales soient orientées non seulement sur l'amélioration de la qualité de vie des personnes âgées, mais aussi sur la rétention des jeunes. Car pour assurer son dynamisme et sa vitalité, un territoire, quel qu'il soit, n'a-t-il pas besoin d'une population aux tranches générationnelles et sociales équilibrées? Le territoire n'est pas seulement un réceptacle, c'est-à-dire un lieu où des événements se produisent. Il est aussi un acteur stratégique, d'où l'importance d'intéresser les élus locaux et régionaux dans le processus de restructuration.

En France, les départements ont le mandat d'élaborer des schémas gérontologiques. Ceux-ci servent à évaluer les besoins des personnes âgées en termes de services de proximité, de loisirs et de logement. En ce qui concerne ce dernier aspect, des formules inédites ont été développées notamment en milieu rural. Par exemple, l'habitat de type kangourou permet à une personne âgée (seule ou en couple) d'occuper le rez-de-chaussée alors qu'un jeune couple (de la famille ou non) habite aux étages supérieurs. Ensuite, la formule «abbeyfield» (c'est-à-dire un habitat groupé participatif) a été créée en Angleterre en 1956 et s'est répandue dans d'autres pays comme la Belgique. Chaque habitant dispose d'un petit appartement complété par des pièces communes qui constituent des lieux de rencontre et d'activités. Ce concept s'adresse notamment aux personnes vieillissantes à revenus modestes qui sont encore autonomes.

Il nous semble qu'au Québec, les MRC auraient avantage à s'inspirer de tels modèles en y intégrant un volet à l'intention des jeunes. La solidarité intergénérationnelle est naturelle et indispensable à la vie d'une société. En ce domaine, certaines municipalités ont mis en place des initiatives visant à répondre spécifiquement aux attentes de ces deux catégories d'individus, mais leurs actions demeurent bien timides eu égard aux besoins à combler et aux moyens dont elles disposent. Pour cette raison, il importe de mettre en place, notamment au sein des milieux fortement enclins à la «gérontocroissance», au vieillissement démographique 
et à l'exode, des mesures à l'intention des MRC et des municipalités locales destinées à encourager l'émergence d'initiatives novatrices en matière de logement social. L'une des stratégies qui nous semblent les plus efficaces est la différenciation : jouer sur les avantages comparatifs propres à chaque milieu et à son histoire particulière. En outre, de telles initiatives n'auraient que des effets positifs sur le dynamisme de l'habitat et, par conséquent, sur l'aménagement du territoire, le développement local ainsi que sur l'amélioration de la qualité de vie des personnes âgées. Les élus sont donc conviés à innover dans la conduite de projets territoriaux où chaque collectivité trace son chemin en fonction d'une identité et de besoins spécifiques. Leurs actions, tout comme celles des instances gouvernementales, doivent s'inscrire dans le sens de la réduction des disparités économiques et des inégalités interindividuelles, une condition essentielle au développement régional et à l'épanouissement plein et entier des personnes et des communautés. 
Pub

ROT site web 